Sciendo

\title{
A Simple Realist Account of the Normativity of Concepts
}

\author{
Elisabetta Lalumera \\ Università degli Studi di Bologna
}

Disputatio Vol. 1, No. 19

November 2005

DOI: $10.2478 /$ disp-2005-0008

ISSN: 0873-626X 


\title{
A simple realist account of the normativity of concepts
}

\author{
Elisabetta Lalumera \\ Università degli Studi di Bologna
}

\begin{abstract}
I argue that a concept is applied correctly when it is applied to the kind of things it is the concept of. Correctness as successful kind-tracking is fulfilling an externally and naturalistically individuated standard. And the normative aspect of concept-application so characterized depends on the relational (non-individualistic) feature of conceptual content. I defend this view against two objections. The first is that norms should provide justifications for action, and the second involves a version of the thesis of indeterminacy of reference.
\end{abstract}

\section{Introduction}

Among the reasons in favour of the claim that concept-application is normative there is the intuition that concepts, just like words, can be applied rightly or wrongly. Concepts are currently identified with mental representations of objects and properties (Fodor 1998a), and/or with mechanisms for picking up, storing and delivering information about objects and properties (Millikan 1999, 2000; Prinz 2002). The intuitive point is that we might fail both in representing things as they are, and in categorizing things as being of a certain kind. If it were not so, then the distinction between appearance - what we take things to be — and reality — what things are — would collapse.

Many philosophers, however, think that normativity is incompatible with semantic naturalism, the view that 'everything that ever happens, including our being rational, is the conformity of nature to law.' ${ }^{1}$ A well-known philosophical argument supports such a convic-

\footnotetext{
${ }^{1}$ Fodor (1998b, 4). I will also group under the label 'Naturalism' the metaphysical thesis that if semantic properties are real, then it must be that they are instantiated in virtue of the instantiation of certain non-semantic properties.
}

Disputatio, Vol. I, No. 19, November 2005 
tion. By means of a sceptical paradox, Kripke argued that no fact nothing that would fall within a naturalistic description of reality can constitute a standard of correctness for the use of words and concepts ${ }^{2}$. Kripke's conclusion is widely accepted, so that normativity is generally dismissed by naturalistically inclined philosophers and cognitive scientists.

In this paper I defend a simple realist view about concept-application that is naturalistically acceptable, while making room for the idea that cognitive performances are subject to standards of objective correctness, i.e., they are normative in a basic sense. I will discuss two objections to simple realism, the second giving raise to different problematic cases. My general strategy will be to suggest that the alleged incompatibility between naturalism and normativity disappears as soon as we move away from sceptical scenarios, and take a closer look at how cognition works in the actual setting of our environment.

\section{A simple realist thesis}

The sketchy model of cognition presupposed by the simple realist thesis can be described as follows. Concepts are detectors and indicators of objective patterns of similarities ${ }^{3}$ — call them 'kinds. ${ }^{.4}$ Applying concepts is basically identifying something as being of a certain kind (as in the thought 'This is a geranium'), or representing something as being of a certain kind (as when I remind to myself that a geranium is a warm-climate plant). ${ }^{5}$ In the former case — identifica-

\footnotetext{
${ }^{2}$ Kripke 1982 . The exception worth noting is the teleological theory of concepts developed in Millikan 2000.

${ }^{3}$ I borrow the terms 'indicators' and 'detectors' from Prinz (2002).

${ }^{4}$ I will use the term 'kind' and the expression 'concept-application' where many (I think) would have used 'category' and 'categorization.' The main reason for this choice is that I often find the talk of categories as being ambiguous between two very different readings ('concepts are categories' versus 'concepts refer to categories').

${ }^{5}$ A further question here would be how concepts represent and identify kinds, i.e. the psychological (engineering) problem of describing the mechanisms of cognition. Though I am aware that some philosophers, and maybe most cognitive scientists, take it to be the problem about concepts (see e.g. Murphy 2002, Harnad 2003), the main concern of this paper allows me to overlook the issue almost completely.
} 
tion, or 'systematic differential response' — the function of a concept is that of a 'mental glue,' as it makes it possible to project stored information about the kind to the newly encountered case. ${ }^{6}$ In the second case — representation — a concept would function as a placeholder of the kind of things it represents, enabling the construction of a model of reality of which that kind of things is an element (a belief, a desire, a plan, etc.). ${ }^{7}$ Though abstractly distinguishable, the two functions of detecting and representing are likely to be interdependent. For example, it is likely that the possibility of identifying the same kind of things among various circumstances, aspects and conditions, is enhanced (among other factors) by the availability of representations of that kind of thing as having variable properties.

Kinds of things are taken here to be objective patterns of similarity, in that things belong to a kind in virtue of the properties they share, over and above the property of being represented by us with the same concept. In other words, kinds are concept-independent, at least in the general case, in that being conceptualized as the same does not suffice for being the same. ${ }^{8}$ Concept-independence of kinds motivates the label 'realism' that can be attached to this picture. The opposite (anti-realist) view would have it that, in the general case, the extension of concepts is determined by people's agreement in applying them.

The Simple Realist thesis about concept-application is that a concept is applied correctly when it is applied to the kind of things it is the concept of - fulfilment of the norm associated with the concept consists in that. So, for example, I apply the concept GERANIUM correctly to the plant I see on my window-sill if and only if that plant is really a geranium. Here, the (natural) relation between my cognitive system and the natural kind geranium provides the standard of correctness for my use, like the (conventional) relation between meter bar in Paris sets the standard of correctness for my measurements

\footnotetext{
${ }^{6}$ The view of concepts as substance-trackers is developed by Millikan (1999, 2000); see also Harnad (2003); Murphy (2002) for the term 'mental glue.'

${ }^{7}$ The view of concepts as building-block of thoughts is explicit in Fodor (1998) and Laurence and Margolis (1999).

${ }^{8}$ This is not yet to say that the kinds of things there are — in short, ontology — does not depend on our interactions with the environment, for example in perception or in action, as I hope will be clear in the next sections. One thing is to say that ontology comes before conceptualization, quite another thing is to say that ontology is completely unconstrained by our cognitive resources.
} 
with the decimal system. In both cases, the second term of the relation is external to the cognitive system, it is out there in the subject's environment. Therefore, correctness of conceptual application as successful kind-tracking is fulfilment of an externally individuated standard of correctness, and the normative aspect of conceptapplication so characterized crucially depends on the relational (nonindividualistic) feature of conceptual content.

The simple realist thesis fits well with the intuition that there can be conceptual mistakes. I will borrow a few lines from Laurence and Margolis to illustrate the point:

When Jane is nervously trekking through the Amazon jungle, fearful of snakes, and she is startled by what she takes to be a snake lying across her path just ahead, we want it to be possible that she could actually be mistaken, that it could turn out that she was startled by a snake-shaped vine, and not a snake at all (Laurence and Margolis 1999, 35).

Now, for it to be possible that Jane is mistaken in her application of the concept SNAKE, we need to make room for a distinction between the class of things that Jane identifies as snakes, and the class of things that are snakes. One straightforward way to make room for such a distinction is by appealing to the idea of concepts as trackers of objective, concept-independent kinds. The natural kind snake is involved in the individuation of the norm according to which Jane's performance with the concept SNAKE can be evaluated as right or wrong. Vice versa, on the view that Jane's mechanisms of application of the concept SNAKE determine the extension of the concept, the above distinction would be lost, and the possibility of describing error would be gone as well. As far as I know this latter position has never been intentionally endorsed. Philosophers usually extract norms out of the performance of ideal subjects in ideal conditions, or out of the performance of a community of subjects. Still, the no-error position is worth noting as a possible pitfall one should be aware of.

The simple-realist thesis is compatible with a broadly naturalistic stance, as kinds, or patterns of similarity, are to be understood as natural properties. ${ }^{9}$ It describes our conceptual capacity as a continuum

\footnotetext{
${ }^{9}$ For a naturalist ontology of kinds, the reference is Millikan 2000, chapter 2. See also Lewis 1983.
} 
with more basic perceptual-recognitional capacities that we probably share with other animals.

Nevertheless, one can accept simple realism and acknowledge, in addition, other levels of normativity within an explanation of people's use of concepts. For example, one may argue that many concepts entertain entailment links one to the others, so that their application can be evaluated according to norms of inferential correctness. This would be particularly evident for logical and mathematical concepts, for theoretical concepts of science, but also for concepts of days of the week, months, and other systems of notation. More levels of normativity of concept-application can be added by considering norms of rationality in a broad sense. The simple realist is just committed to claiming that concept-application can be evaluated at least because it is constrained by the nature of what concepts are of.

\section{First objection. The justification requirement}

A reaction that the simple realist thesis may trigger is that kinds cannot provide the standard of correctness for the application of concepts, because the extension of a natural kind - even of the most familiar of them — is generally beyond the knowledge of average subjects, and therefore kinds cannot be used as a standard, in the sense that they cannot guide nor justify the subject's use of the corresponding concept.

The second step of the objection - the epistemic remoteness of kinds - is usually backed by two familiar considerations. First, it is a fact that we usually ignore intrinsic properties of most natural kinds, i.e. we do not possess complete knowledge of intensions - or whatever determines extensions of kinds. For example, one may know what mercury looks like and what it is used for, while being uncertain about who to defer to for more information and correct recognitional judgments. The intension of the natural kind mercury falls out of the epistemic reach of such an individual, and does not guide her use of the corresponding concept - it provides by no means a standard she can confront with. A similar kind of thought is developed by Fodor (1998a, 154-163), who argues that natural kinds are late and highly reflective products of human science and reflection, and therefore cannot be what everyday concepts refer to - appearances came first, and should come first when it comes to ordinary concepts. 
The second point about the epistemic remoteness of kinds is that extensions of kinds are beyond one's epistemic reach. A natural kind, intuitively, somehow contains the whole class of things that belong to that kind, even though they are scattered in space and time. The extension of one's concept GOLD contains tons of gold. No doubt that an epistemic connection with such an entity is problematic. This was basically Wittgenstein's point with the rules-as-rails metaphor: no infinite entity, such as the range of values of a function, can be the standard of correctness for our application of rules, concepts, and words, because we lack appropriate epistemic access to infinite entities (Wittgenstein 1953, §§ 186, 195-197).

I will not discuss the epistemic remoteness of kinds here, as the discussion would require a deeper investigation on the metaphysical status of kinds, which would far exceed the limits of this paper. In fact, I think that the second step of the objection can be accepted without dismissing the simple realist thesis. It may well be that our knowledge of kinds and of their intrinsic properties is scarce, and plays a minor role as a mechanism of identification of specific instances. Still, objective patterns of similarity can play the same role with respect to concepts as the meter bar with respect of my measurements - I have never seen the meter bar, I could even be unaware of its existence, but when I pick up the pocket meter and measure the distance between my desk and the wall, my measurement can be assessed as correct or incorrect according to that quantity (the meter bar) as a standard. Correctness and incorrectness of a measurement judgment are objective matters insofar as the meter bar exists, but independently of my epistemic access to it. Likewise, on the hypothesis that concepts track kinds, correctness of concept-application is an objective matter, independently on one's knowledge of the extension of the kind.

This is, however, precisely what the objection denies. The objection explicitly endorses the claim that norms, and a fortiori norms for cognition, should guide and justify one's response to the stimulations from the environment. Such a justification requirement on candidate conceptual norms plays a central role in Kripke's discussion of the skeptical paradox about meaning:

An answer to the sceptic must satisfy two conditions. First, it must give an account of what fact it is (about my mental state) that constitutes my meaning plus, not quus. But further, there is a condition that any putative 
candidate for such a fact must satisfy. It must, in some sense, show how I am justified in giving the answer ' 125 ' to ' $68+57$ ' ( Kripke 1982, 11).

But for the conclusion to follow — that kinds cannot be conceptual norms - a further step is needed, that is, the idea that justifications must be epistemically accessible from the first-person viewpoint. The missing step would have the form of an Accessibility Condition:

Some content c constitutes a subject's justification for believing $P$ if and only if c is conceptually accessible to the subject.

The Accessibility Condition is far from being unobjectionable. As it is well-known, it is opposed by externalist accounts of knowledge, both of reliabilist and of more moderate kinds. According to classical reliabilism someone's beliefs are justified insofar as they are outputs of a reliable process, that is, a successful process, whose outputs are truths or veridical contents (Goldman 1979; Alston 1995). Existence and functioning of the process need not be accessible by the individual whose epistemic performance is under assessment.

It is well known, however, that classical reliabilism is vulnerable to counterexamples, which would undermine the plausibility of its basic insight — cases in which our pre-theoretical intuitions on the notion of warrant would preclude the application of such concepts to outputs of truth-generating process. In fact, reliabilism in its classical form ca not exclude from reliable processes series of casual (but repeated) truth-matching, and mechanisms that have nothing to do with the properties or attitudes of the person or living being in question. In such cases, the route to veridicality or knowledge would be not only conceptually or rationally inaccessible to the subject — as according to a basic externalist attitude — but also completely independent from the subject's intrinsic and relational properties. On the assumption that epistemology is a (philosophical) explanation of how a subject gains knowledge, there are reasons for concluding that classical reliabilism, insofar as it allows for the above-mentioned cases, can be blamed for changing the subject altogether.

A more moderate externalist strategy consists in dropping the Accessibility Condition while preserving the idea that what matters to the epistemic status of a subject's claims are processes and mechanisms that depend at least in part on her intrinsic and relational 
properties, and therefore can explain her route to knowledge. ${ }^{10}$ According to Burge 2003, the condition of proper functioning of a subject's cognitive sub-systems provides genuine warrant (entitlement in his terminology) to categorical judgments. Conditions of proper functioning are individuated given the function of a system and its goal, or epistemic good - in the case of representational systems (like the perceptual and the conceptual system), representing is the function, and veridicality and truth are the epistemic goods. Given that the function of each concept is to track and represent what it is the concept of, successful kind-tracking provides a warrant for judgments of correctness of cognitive performance. The existence of such a warrant is both dependent on the characteristics of the system or the organism, and on external environmental features, thereby preserving the externalist insight as well as the explanatory constraint.

If an externalist view of warrant such as Burge's is adopted, it can be concluded that the objection of the Justification Requirement fails against the simple realist thesis, because it involves an internalist conception of knowledge, which is assumed without a sufficient case. The objection is nevertheless right in pointing out that conceptual correctness as successful tracking of a kind requires a strongly objective, and epistemically independent account of normativity and correctness. On the simple realist view of normativity, norms are out there and not within the range of the subject's introspection; they need not be understood or intentionally adhered to by the subject they apply to. In a clear sense, they do not guide concept-application, but they are fulfilled, or not fulfilled, from a purely objective epistemic standpoint. ${ }^{11}$

\section{Second objection. The which-question}

The most serious difficulty arising in connection with the simple realist thesis can be condensed in a question: Which kind of things sets the standard of correctness in a given case of concept-application, of

${ }^{10}$ According to Burge, 'whether an epistemic state is warranted depends not only on whether it provides, in a certain sense, a good route to truth. The notion of epistemic warrant is also conceptually tied to considerations regarding limitations of perspective, information and structure in the particular system or animal at hand' (Burge 2003, 5).

${ }^{11}$ See Burge 2003 for this kind of view. 
the many possible ones that can be identified? Not only are all things the members of an infinite number of different kinds, but each of their properties, and combination of properties is a potential basis for assigning them to still more kinds. So when I look at the window-sill and think that I will move the geranium to the balcony, which is the natural kind I am keeping track of - geranium, plant, that particular individual I bought in April, or any red-green-and brown object? And therefore, according to which standard can my application of the concept GERANIUM can be said to be correct? Kripke's skeptical paradox about meaning can be seen as a dramatization of such a worry. In Kripke's own example, a table seen at the entrance of the Eiffel tower belongs to the kind table, but also to many different and more or less gerrymandered classes of things, for example, the classes of things seen at the entrance of the Eiffel tower. So, a skeptic could argue for this purely logical possibility: that my concept TABLE as I used it up to now has always tracked the things that are tables but are not seen at the entrance of the Eiffel tower. The skeptic and I would therefore disagree over the correctness of the judgment 'This is a table' when applied to a table at the entrance of the Eiffel tower, as I would take it to be correct, and the skeptic would not. But we cannot be both right. The concept cannot track either one kind or the other - especially when the alternatives are infinite as a logical possibility, as Kripke's skeptic suggests. Such a scenario - in fact, a skeptical scenario would deprive the notion of correctness, and of meaning, of any stability. Thereby, the need of giving reasons for choosing which is the intended pattern, among the various alternatives.

Notice, also, that talking of patterns of similarity does not help here. Even without following Goodman's thought that similarity is a purely projective matter, it can be proved that, given any pair of objects, there are almost infinite respects in which they are similar, from a purely logical point of view. So, strictly speaking (and skeptically speaking) the ugly duckling of Andersen's story is no less similar to his step-brothers, than they resemble one another (Watanabe 1985). And grue things are similar to one another like green things are. The only way out is to single out some aspects of similarity, and give them more weight. But this is precisely the problem.

The point of the objection, in a nutshell, is that the simple realist thesis would underdetermine the question of correctness - there are too many kinds out there for them to count as norms for the use of concepts, even in the purely objective, epistemically uncommitted 
sense mentioned above. The rest of this paper will be dedicated to suggest that it may not be the case, that is, kinds are not too many after all, when appropriate constraints are placed. So the objection is no knock-down argument for the simple realist.

\section{Constraints}

The simple realist's strategy diverges from well-known reactions to the Which-question. Usually, solutions to the Which-question take the form of refining one's account of the relation between concepts and what they refer to. Fodor's Asymmetric Dependence Theory, and Millikan's teleological theory share this form of solution to the Which-question. ${ }^{12}$ Rather than trying to individuate the error-proof mind-world correlation, the simple realist's strategy concentrates on placing constraints on which kinds of things there are for us to conceptualize, that is, which are the kinds of things that our cognitive system would afford to represent. ${ }^{13}$ If we admit that for any single case of concept-application the possible standards of correctness are infinite, and all of them are on par, then it is like having no standard of correctness at all. However, we are not forced to accept the antecedent, as far as constraints can be placed in order to restrict the range of possible concept-individuating kinds, with the effect of varying the weight of possible alternatives. In fact, the general strategy of constraints goes back to David Lewis $(1983 ; 1984)$, who proposed to solve the problems of indeterminacy of reference (Quine's problem and Putnam's paradox) by putting constraints on what an eligible referent can be. Consider this passage:

\footnotetext{
${ }^{12}$ In Fodor's case, the general view is that all concepts track kinds via causal connections - as kinds are reliable causes of concepts, concepts carry information about kinds. Even though typically each concept tends to carry information about too many kinds, only a few of these causal-informational connections are to be taken as fundamental and meaning-giving. Same attitude, but different solution, can be attributed to Millikan. Millikan suggests that the Which-question be approached by adding a historical constraint to the concept-kind causal. I will not discuss any of the two proposals here - whether any of them could provide successful responses to the objection raised above, or not, I think that a different strategy might be worth exploring.

${ }^{13}$ Affordance is a concept from Gibson's study of perception (Gibson 1979).
} 
I would instead propose that the saving constraint concerns the referent — not the referrer, and not the causal channels between the two. It takes two to make a reference, and we will not find the constraint if we look for it always on the wrong side of the relationship. Reference consists in part of what we do in language or thought when we refer, but in part it consists in eligibility of the referent (Lewis 1983, 220).

A well-known objection to the general project needs to be mentioned before starting. One could maintain that if reference is generally indeterminate, then so is the reference of the terms used to lay down the relevant constraints - so what entitles me to the view that in laying down certain constraints, I have achieved anything determinate $?^{14}$ In other words, why should the metalanguage of the constraints be meaningful at all? The objection amounts to the move of a prototypical meaning-sceptic, who silences any opponent's claim on the grounds that to presume them meaningful is to beg the question. A short and clear formulation of an answer available to the realist is reported by Hale and Wright, elaborating from Lewis:

Obviously the metaphysical realist has to be presumed capable of contentful — so, determinately referential — speech, if he is to respond to ... any challenge at all. The onus legitimately placed upon him is not to demonstrate that determinate reference is possible, but to provide a constitutive account which explains how determinate reference works. Accordingly, he is perfectly within his rights to assume, at least pro tem, a metalanguage in which a determinate account of the putative mechanics can in principle be given (Hale and Wright 1997, 441).

\section{Perception, ontology and action}

The constraints proposed by the simple realist in order to answer the Which-question may be grouped into three broad and partially overlapping categories, by considering their origin.

${ }^{14}$ I owe this formulation of the 'More theory objection' to an anonymous referee of Disputatio. 


\section{From perception}

Similarity is first of all detected by our sensory system, which has (so to say) preferences, and default values. Consider the case of colors. We are equipped to detect colors through light stimulation, when it occurs within a certain wavelength (we are blind to frequencies above red, about $800 \mathrm{~nm}$, and below violet, about $400 \mathrm{~nm}$ ). Within such boundary, however, we do not see each value as a different color. Specialized detectors sort out different incoming frequencies into broader groups, so that the outcome of different frequency values is vision of the same color. So in normal conditions, some similarities are enhanced, other inhibited. The result of this complicate process is the selected range of patterns of similarities that we may track with color concepts.

More examples can be obtained by considering invariancedetectors mechanisms. We are equipped to track the invariance of a three-dimensional shape through motion, and translate it into visual constancy — we see the same object as the same again, before, during and after its motion, in a different place. Again, in this case, our sensory system gives different weights to the object's features, selecting out a few of them as a basis for re-identification. As a result, normally we do not sort things out into moving objects versus still objects - though it would still be metaphysically possible for us to do so. For example, I call my dog Pierre both when he is running, and when he is sleeping on the carpet; I see the geranium as the same plant on the window-sill and on the balcony, and so on.

Moreover, our sensory system tends to give representational advantage to dense and self connected objects, with respect to classes of scattered parts - an object is self connected when given any two parts of it, there is a path joining them that never travels outside the boundaries of the object. ${ }^{15}$ Such objects tend to have projectible properties, and they are more easily connected with basic practical functions of our organism, such as grasping, eating, avoiding, and mating. In this sense, Quine's undetached rabbit parts are worse objects than rabbits, and rabbits are far more eligible referents for the GAVAGAI concept than their skeptical alternatives.

In very general terms, categorical perception (what we can do with a sensory system and invariance detectors) enhances some

\footnotetext{
${ }^{15}$ See for example Bloom (1996, 90); Spelke 1994.
} 
patterns of similarity and ignores others, so that even though a thing belongs to an infinite number of kinds, only a selected subset of them can be successfully tracked by our representational systems, given their function and architecture, and a subset of that subset would be tracked in a normal environment as a default or preferred option (Harnad 1987; 2003). Thereby, categorical perception puts constraints on what there is for us to detect and represent with concepts. As soon as we consider the actual situation of cognition, the logically equivalent alternatives of a skeptical scenario are no more on par, ceteris paribus — some of them fall beyond the metaphysical possibility of what we can represent, while some others are given a low degree of preference. As an example of the latter kind, it is certainly true that we may happen to succeed in tracking, say, a grue pattern when we apply GREEN, and we are able to represent grue things by means of a concept - in fact, philosophers do. But an unsupervised learning machine equipped with our same sensory system after a sufficient period of experience would sort out the green kind in normal circumstances, as it conforms to its preference values and filters; the machine would presumably derive a detection procedure for the grue pattern which is parasitic on the green-detection mechanism. This is sufficient for claiming that the two alternative patterns of objective similarity have different weights for a representational system, and that one can be singled out as the default value. Similarly, in normal conditions the class of things that are tables but not at the entrance of the Eiffel tower is not the default semantic value for the mental representation TABLE, neither are undetached rabbit parts for rabbit, when compared to rabbits. ${ }^{16}$

\section{From ontology}

The simple realist may even take one further step. She may want to add that the range of eligible referents is constrained by the very nature of our environment, and not only by the nature of our representational system. Eligible referents are the natural objects and kinds sciences deal with — where 'science' is taken broadly as meaning any systematic explanation of the characteristics of our environment by providing predictive laws, or law-like generalizations. The characteris-

\footnotetext{
${ }^{16}$ See Leonardi 2003 for perceptual constraints applied to Quine's indeterminacy problem.
} 
tics of our environment are explained in terms of (say) green and rabbits, not in terms of grue or undetached rabbit parts, as such deviant entities do not play any role in the biological and physical life of our world (undetached rabbit parts do not breed, and grue objects do not possess surface light-reflectance properties in common). The Which-objection, especially in its skeptical version, assumes that eligible referents or standard of correctness for concepts are far more problematic than natural kinds. But an externalist and naturalist position such as the simple realist's can refuse such an assumption, by opposing the view that what is out there in the actual world affects the content of our minds at least as much as the characteristics of our mind affect our access to reality.

\section{From intentional action}

Constraints coming from categorical perception and ontology provide an answer to the Which-question in skeptical scenarios; they are not sufficient, however, when more ordinary cases of underdetermination are involved. What I see on my window-sill may not be a grue thing, but it is still, at the same time, a geranium, a plant, an object, a living object, these being perfectly eligible kinds from the point of view of perception and explanatory value. So which one's in charge of providing the norm for my conceptual judgment? In such a case, kinds are not infinite, but they are still too many.

Another hint from cognitive psychology might rescue the simple realist here. The idea is that concept-application does not occur in the void, or, which is equivalent, in the pure space of philosophical reflection. We normally interact with the environment in order to do something, or to extract some specific piece of information, that is, we move cognitively in a goal-oriented way. So, for example, I may interact with the geranium in order to grasp it, to smell it, to make the inventory of my personal belongings, to relax my eyes with a greenish visual image, to check whether I should do some watering, etc. Though it remains true that the geranium belongs to many different and perfectly objective kinds, far less of these kinds are salient or relevant when it comes to a given episode of cognitive contact. In each occasion, there may be just one kind of thing that I aim at tracking and representing, and according to which my thoughts and actions can be evaluated as true or false, successful or unsuccessful. Cognitive 
scientists who work within the paradigm of embodied cognition may draw the philosophical conclusion as follows:

The aboutness of the representational format of our mind is deeply rooted in the intrinsic relational character of body action, which, in turn, suggests the intrinsic intertwined character of action, perception and cognition (Gallese 2000, 78).

Notice that simple realism does not go in the direction of maintaining that ontology is determined by human values and preferences $;{ }^{17}$ as specified in point 2 above, it rather claims that what there is remains largely independent of human cognition, but also acknowledges that problems of indetermination of reference in given episodes of concept-application (that is, the problem of what we choose to represent and why) can be solved by taking into account cognitive goals.

The additional constraint on the range of eligible referents suggested here is the element of purpose, which enters the picture of concept-application both in the teleological sense favored by Millikan (each concept is formed and preserved with the aim of keeping track of a specific kind of things), and in the sense that most cognitive interactions, and a fortiori most concept-applications, display a means-end structure. If categorization tasks are goal-sensitive, normative standards are goal-sensitive, too.

\section{Conclusion}

I have claimed that vis-à-vis the Justification Requirement and the Which-question objection, simple realism possesses the resources for a reply. Concepts, as trackers of natural kinds, are norm-governed in that they are subject to standards of correctness provided by the kinds themselves. Our cognitive activity can be evaluated with respect to them from an objective point of view; nevertheless, the sensitivity of our cognitive system to objective patterns of similarity does not guide our concept-applications as a set of explicit rules would do. The sceptic's claim that objective standards so conceived are infinite for any episode of concept-application, and therefore useless, has been rejected by pointing to a number of constraints on what a human

\footnotetext{
${ }^{17}$ For an idealist view of this kind see Rosch, E., Thompson, E., Varela, F., 1991.
} 
cognitive system can afford to represent. With this move, simple realism as a thesis on concept-application gains the status of a hypothesis, because it rests on a posteriori grounds; it depends on certain facts about our minds and the environment being true. The conclusion is not yet that the indeterminacy problem posed by the sceptic disappears, but rather that the problem of conceptual correctness is sized down to an empirical problem for cognitive agents in an actual environment. ${ }^{18}$

Elisabetta Lalumera Università degli studi di Bologna Dipartimento di discipline della comunicazione

Via Azzo Gardino, 23

40122 Bologna, ITALY lalumera@dsc.unibo.it

\section{References}

Alston, William. 1995. How to Think about Reliability. Philosophical Topics 23. Bloom, Paul. 2000. How Children Learn the Meaning of Words. Cambridge, (Mass.): MIT Press.

Fodor, Jerry. 1998a. Concepts: Where Cognitive Science Went Wrong. Oxford: Oxford University Press.

. 1998b. In Critical Condition. Oxford: Oxford University Press.

Gallese, Vittorio. 2000. The Inner Sense of Action: Agency and Motor Representations. Consciousness Studies 7, no. 10.

Goldman, Alvin. 1979. What is Justified Belief? In G. Pappas (ed.), Justification and Knowledge. Reidel: Kluwer Academic Publisher.

Goodman, Nelson. 1983. Fact, Fiction, and Forecast. London: Athlone Press, $1954,4^{\text {th }}$ ed., Cambridge, (Mass.): Harvard University Press.

Gibson, James. 1979. The Ecological Approach to Visual Perception. Hillsdale (NJ): Lawrence Erlbaum.

Hale, Bob and Wright, Crispin (eds.). 1997. Putnam's Model-Theoretic Argument. In A Companion to the Philosophy of Language. Oxford: Blackwell.

${ }^{18}$ My greatest debt is to Paolo Leonardi for initial discussion of these issues. I also wish to thank the participants of the Workshop on Concepts held in October 2003 at the University of Navarra (Pamplona). I am grateful to Tyler Burge for comments on an earlier version of this paper, and to an anonymous reviewer at this journal. 
Harnad, Stevan. 1987. Category Induction and Representation. In Harnad, S. (ed.) Categorical Perception: The Groundwork of Cognition. New York: Cambridge University Press, 1987.

. 2003. Categorical Perception. In Encyclopedia of Cognitive Science. London: Macmillan.

Kripke, Saul. 1982. Wittgenstein on Rules and Private Language. Oxford: Blackwell. Laurence, Steven. and Margolis, Eric. 1999. Concepts and Cognitive Science. In Concepts: Core Readings. Cambridge (Mass.): MIT Press.

Leonardi, Paolo. 2003. Objects of Reference. In Bottani, Andrea e Bianchi, Claudia (eds). Significato e Ontologia, Milano: Franco Angeli.

Lewis, David. 1983. New Work for a Theory of Universals. Australasian Journal of Philosophy 61.

1984. Putnam's Paradox. Australasian Journal of Philosophy 3.

Millikan, Ruth. 1999. A Common Structure for Concepts of Individuals, Substances and Real Kinds. More Mama, More Milk and More Mouse. Brain and Behavioural Sciences, 21-23.

2000. On Clear and Confused Ideas: An Essay on Substance Concepts. Cambridge (UK): Cambridge University Press.

Murphy, Gregory. 2002. The Big Book of Concepts. Cambridge (Mass.): MIT Press.

Prinz, Jesse. 2002. Furnishing the Mind: Concepts and Their Perceptual Basis. Cambridge (Mass.): MIT Press.

Spelke, Elizabeth. 1994. Initial Knowledge: Six Suggestions. Cognition 50.

Rosch, Eleanor, Thompson, Evan, Varela, Francisco. 1991. The Embodied Mind: Cognitive Science and Human Experience. Cambridge (Mass.): MIT Press.

Wittgenstein, Ludwig. 1953. Philosophical Investigations. Oxford: Blackwell. 